\title{
Análisis de la onda de presión arterial en Anestesiología y Cuidados Intensivos I.
}

Paz Martín D.

Complejo Hospitalario de Toledo.

\section{Resumen}

La frecuencia y la forma del pulso arterial han sido empleadas desde hace milenios en un amplio abanico de escenarios clínicos.

Cada componente de la onda de la presión arterial; presión pico, presión diastólica, tiempo de eyección, ascenso de la presión arterial durante la sístole y presión arterial media son el resultado de una compleja interrelación de procesos ventrículo-arteriales. Su correcta interpretación puede ser de gran utilidad a la hora de tomar decisiones clínicas.

\section{Introducción}

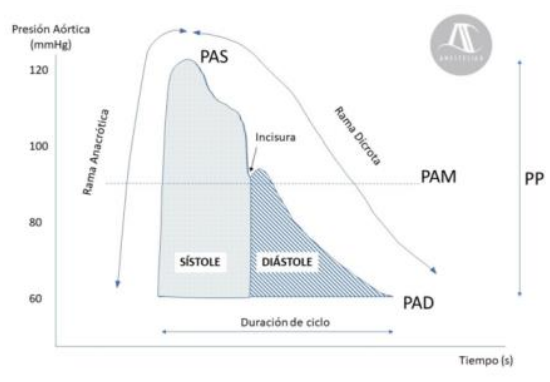

La frecuencia y la forma del pulso arterial han sido empleadas desde hace milenios en un amplio abanico de escenarios clínicos.

Cada componente de la onda de la presión arterial; presión pico, presión diastólica, tiempo de eyección, ascenso de la presión arterial durante la sístole y presión arterial media son el resultado de una compleja interrelación de procesos ventrículo-arteriales. $\mathrm{Su}$ correcta interpretación puede ser de gran utilidad a la hora de tomar decisiones clínicas.

\section{Aspectos técnicos de la monitorización de la presión arterial}

La monitorización invasiva de la presión arterial requiere la inserción de un catéter que se conecta a un sistema de traducción de presión. Tanto el catéter como los tubos del sistema deben estar fabricados en materiales semirrígidos que no absorban la presión que debe llegar al transductor. Estos sistemas habitualmente contienen un sistema de arrastre de suero salino heparinizado y se presurizan a $>200$ mmHg para permitir un flujo de unos 2$4 \mathrm{ml} / \mathrm{h}$ a través de la punta del catéter arterial, que prevenga la formación de microtrombos que alteren la medición.

La presión de la columna de salino dentro del tubo varía con la presión de pulso arterial, lo que produce una deformidad de la cúpula del diafragma del transductor y esto a su vez, un cambio proporcional en la resistencia de un circuito eléctrico tipo puente de Wheatstone. Esta señal es amplificada y procesada por un monitor y el contorno de la onda arterial aparece dibujado por 
un osciloscopio, acompañado de una lectura digital.

Debido a que la arteria radial es superficial y presenta circulación colateral, lo que disminuye la probabilidad de complicaciones isquémicas, es la arteria habitualmente empleada para monitorizar la presión arterial invasiva. Mediante el test de Allen se puede valorar la presencia de circulación colateral y disminuir aún más la probabilidad de isquemia; para realizarlo, con la mano elevada, se solicita al paciente que apriete el puño mientras se sujeta la muñeca del paciente con ambas manos ocluyendo las arterias radial y cubital durante $30 \mathrm{~s}$. A continuación, con la mano aún elevada se solicita al paciente que la abra. La mano y las uñas deberían encontrarse pálidas. Por último, tras liberar la presión de la arteria cubital mientras se mantiene la presión radial debería recuperarse la coloración cutánea a los 5-10 segundos permitiendo de esta forma verificar la permeabilidad del arco palmar.

Cero de presión: el transductor debe exponerse a la atmósfera para calibrar el 0 a nivel del $4^{\circ}$ espacio intercostal en la línea medio axilar (eje flebostático). Este es un aspecto al que a menudo no se da la importancia que tiene; si el transductor se encuentra $10 \mathrm{~cm}$ por debajo de este eje, la lectura de la presión arterial estará erróneamente elevada $10 \mathrm{cmH} 2 \mathrm{O}$ o $7,5 \mathrm{mmHg}$. Se debe realizar el 0 de presión arterial cada 8 horas o cuando se sospeche que la medición es errónea.

\section{Bases fisiológicas del análisis de la onda arterial}

Según la Ley de Conservación de Masas, en estado de equilibrio la cantidad de fluido que entra en un vaso debe ser igual a la cantidad que sale de él. Por otra parte, la distensibilidad de un vaso afecta al flujo que pasa a través de él de una forma predecible. En 1989 Otto Frank publicó una fórmula matemática que aunaba dentro de la hemodinamia la Ley de la Conservación de Masas y la rigidez vascular.

\section{Efecto Windkessel}

Durante cada contracción cardiaca, una cantidad de sangre es bombeada desde el corazón a la circulación periférica. El sistema cardiovascular, específicamente la aorta y las grandes arterias, gracias a sus propiedades viscoelásticas, convierte la onda de presión sinusoidal generada por el corazón (flujo intermitente) en un flujo y presión constante para los tejidos. Durante la sístole cardiaca, el ventrículo izquierdo eyecta unos $70 \mathrm{ml}$ de sangre a la aorta, a este movimiento longitudinal de la sangre se oponen las resistencias periféricas. En cierta manera la cantidad de sangre expulsada es mayor de la que permite la resistencia periférica localizada en las arteriolas distales y la complianza aórtica absorbe parte de este exceso de volumen. Mediante un desplazamiento transversal, las paredes elásticas de la aorta se expanden para acomodar el volumen sistólico. De esta forma se consigue moderar el incremento de la presión intraarterial a la vez que se transforma la energía cinética de la sangre en energía potencial en la pared de la aorta estirada (Fig.1).

Durante la diástole, cuando se produce el cierre de la válvula aórtica, la presión en el interior de los vasos disminuye lo que produce un acortamiento de las fibras aórticas, y permite convertir de nuevo la energía almacenada en energía cinética manteniéndose el flujo de sangre durante la diástole. De esta forma, la aorta se comporta como una bomba diastólica. 
Con la edad, se produce una degeneración de la elastina de la pared aórtica. La aorta se vuelve menos distensible y disminuye su capacidad de acomodar volumen sistólico sin producirse incrementos de presión, a la vez que se altera su función como bomba diastólica. Este fenómeno tiene 3 consecuencias:

1. Incremento de la presión sistólica.

2. Descenso de la presión diastólica.

3. Incremento de la presión de perfusión.

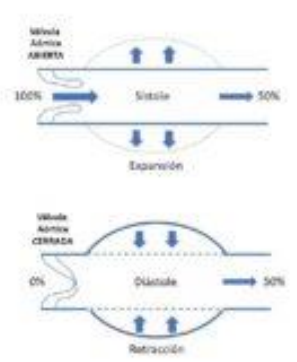

Fig. 1 Efecto Windkessel

Durante la eyección ventricular en la aorta se genera una onda de presión arterial y una onda de flujo sanguíneo.

- Onda de presión arterial: producida por la distensión de las paredes de la aorta durante la sístole y que se propaga a una velocidad en torno a $4 \mathrm{~m} / \mathrm{s}$. Es esta presión arterial la que se palpa como "pulso radial", y no la onda de flujo que se desarrolla más abajo. Conviene, asi mismo, recordar que la velocidad a la que el pulso viaja por la aorta está determinada por la distensibilidad del sistema arterial. Las condiciones asociadas a una disminución de la complianza (ej. vasoconstricción, hipertensión arterial) producen una conducción más rápida de la onda de pulso.
- Onda de flujo sanguíneo: más lenta con una velocidad de avance de unos $20 \mathrm{~cm} / \mathrm{s}$.

\section{Onda de presión aórtica}

Como se muestra en la Figura 2, entre los componentes de la presión arterial intraaórtica encontramos:

Rama Anacrótica (del griego ana, hacia arriba, y crotos, latido). Ascenso pronunciado desde la línea de base hasta un valor pico que corresponde a la presión arterial sistólica. Esta fase comienza con la apertura de la válvula aórtica, coincide con la eyección ventricular y presenta una duración de unos $100 \mathrm{~ms}$.

Rama Dícrota (del griego dikrotos, doble pulsación)

- Un descenso progresivo que corresponde a la parte final de la sístole y al inicio de la relajación ventricular. En este momento el volumen eyectado desde el corazón es mínimo y el flujo de sangre se mantiene gracias al reservorio del compartimento arterial.

- Incisura dícrota: de forma abrupta que interrumpe la pendiente descendente de la presión arterial y representa el cierre de la válvula aortica.

- Descenso posterior hasta la parte más baja de la onda que refleja la presión arterial diastólica.

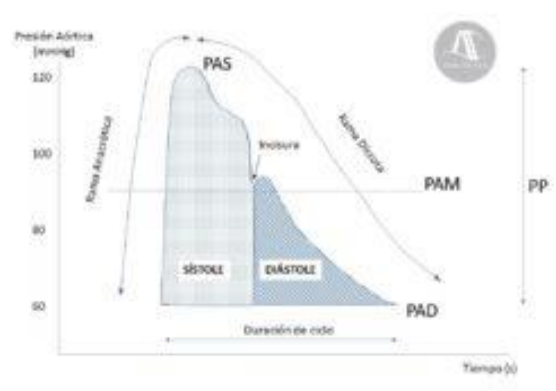

Fig. 2 Onda de Presión Aórtica. PAD Presión arterial diastólica; PAM Presión arterial media: 
PAS Presión arterial sistólica; PP Presión de pulso.

\section{Onda de presión radial}

El físico francés Jean Baptiste Fourier (1786-1830) demostró que las ondas periódicas complejas se pueden expresar como la suma de ondas sinusoidales más simples o armónicos, cada una con su propia frecuencia y amplitud. La morfología de la onda arterial se ve afectada por las ondas de reflexión que se suman a la onda sistólica primaria.

La existencia de ondas de reflexión es típica de los sistemas hidrodinámicos. La onda hacia adelante (onda centrifuga) generada por la bomba intermitente viaja por el árbol arterial y en determinados puntos de reflexión como bifurcaciones arteriales, placas ateroscleróticas y arteriolas terminales, genera ondas que vuelven hacia atrás (ondas centrípetas). Así pues, la morfología de la presión de la arteria radial es el resultado de las ondas de presión que avanzan desde el corazón (y que portan información de la función cardiaca) y las ondas de reflexión que contienen información de los sitios de reflexión y de la periferia del sistema arterial. De esta forma, la presión medida a nivel periférico se encuentra cada vez más distorsionada debido a la trasmisión de una señal en interacción continua con un sistema arterial no uniforme.

Determinadas condiciones patológicas que alteran el tono vascular y la viscosidad, afectan a la amplitud $y$ duración de las ondas de reflexión por lo que raramente las presiones medibles a nivel periférico son iguales a las obtenidas a nivel central.

A menudo la onda de la arteria radial tiene 3 picos (Fig.3) que resultan de los componentes de avance y de retroceso (ondas de reflexión) (1).
PICO 1: tras la apertura de la válvula aórtica que corresponde a la sístole y refleja la eyección sanguínea durante la sístole cardiaca. La presión sistólica está determinada por el patrón y duración de la eyección del ventrículo izquierdo (VI), la distensibilidad de los vasos arteriales, la velocidad de la onda de presión en las grandes arterias y el tono vasomotor a nivel de las arterias periféricas que es responsable de la magnitud de las ondas de reflexión (2).

El incremento de la conducción de las ondas de presión (ej. aumento de la rigidez arterial con la edad) produce un retorno de la onda reflejada a nivel de la unión arterio-arteriolar que se une al siguiente pico sistólico, produciendo una magnificación de la presión sistólica (3).

PICO 2 y 3 relacionados con los sitios de reflexión en la región de las arterias renales y por debajo de la bifurcación de las arterias iliacas. Habitualmente se producen depresiones en forma de valle entre estos picos que pueden denominarse erróneamente muescas dícrotas. Estas depresiones a nivel periférico son característicamente más suaves que en aorta central y guardarían, en el mejor de los casos, una relación residual con la válvula aórtica (Fig. 3)

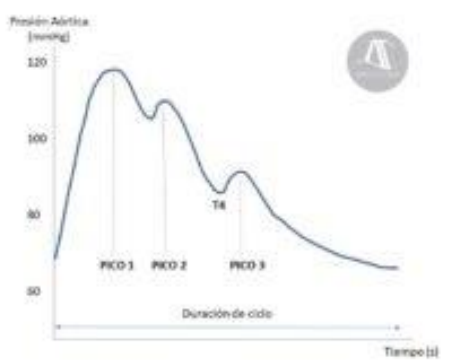

Fig. 3 Onda de presión arterial radial.

Como se indica en la Figura 3, T4 corresponde al tiempo hasta la "muesca dícrota" y coincide con el inicio de la diástole. La parte final de la curva, la PAD, depende de la distensibilidad arterial, la frecuencia cardiaca y la 
resistencia y distribución de la red vascular.

\section{Amplificación sistólica del pulso distal}

Por el mecanismo anteriormente descrito de las ondas de reflexión, se produce una variación de la onda de presión arterial conforme se desplaza distalmente la zona de medición (Fig.4). Los componentes de alta frecuencia como la onda dícrota desaparecen debido a los cambios en la impedancia y a la resonancia de armónicos $\mathrm{y}$, de forma general, se produce un incremento de la PAS con un ascenso más pronunciado y una disminución de la PAD con una muesca dícrota más tardía y menos afilada (esta última no debida al cierre de la válvula aórtica sino a las ondas de reflexión). La PAM en cambio, permanece relativamente constante con independencia del lugar de medición.

Resulta indispensable reseñar que debería considerarse al fenómeno de amplificación distal, no como un error al alza de la verdadera presión de perfusión, sino como un maravilloso mecanismo de eficiencia cardiovascular. Gracias a este fenómeno se alcanzan presiones de perfusión a nivel periférico mayores que a nivel central, consiguiéndose una presión de perfusión óptima con una menor postcarga, y por tanto carga de trabajo, para el miocardio.

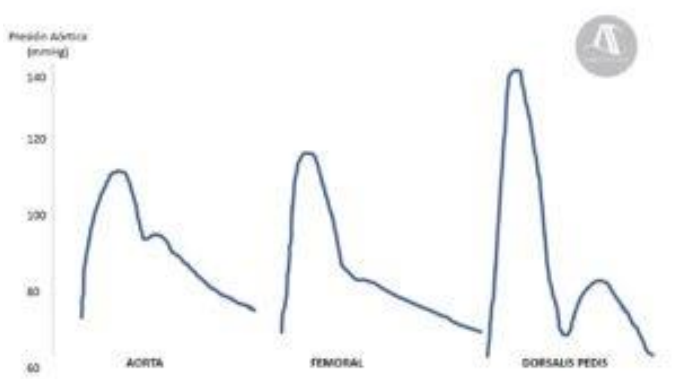

Fig. 4 Modificaciones de la onda de presión arterial según localización de monitorización.
La presión arterial media (PAM) es el valor medio de presión durante el ciclo de pulso arterial, y parece ser el mejor determinante de la perfusión orgánica (excepto para el corazón, que no se perfunde durante la contracción cardiaca, por lo que la presión de perfusión coronaria se aproxima a la presión arterial diastólica en la raíz aórtica). Además, como hay relativamente poca resistencia en el árbol arterial hasta llegar a los vasos más pequeños, se observa sólo una pequeña caída de los niveles de PAM a lo largo de los diferentes puntos de medida.

El valor ofrecido por los monitores integraría el trazado de la onda arterial a lo largo del tiempo y sería mucho más exacto que la determinación con la fórmula clásica

$\mathrm{PAM}=(\mathrm{PAS} / 3+[2 \times \mathrm{PAD}] / 3)$

La PAM depende de 2 factores:

1. Gasto cardiaco (GC): el flujo de sangre que atraviesa el vaso

2. Resistencia vascular sistémica (RVS)

Los factores anteriores se interrelacionan en la siguiente fórmula

$\mathrm{PAM}=\mathrm{GC} \times \mathrm{RVS}$

Presión de pulso

La presión de pulso (PP) es la diferencia entre la PAS y la PAD. Es precisamente la PP y no la PAS la principal responsable del pulso que se palpa. Los dos factores más importantes que podrían aumentar la presión de pulso serían:

1. Incremento del volumen sistólico: todo lo que aumente el volumen sistólico, como una disminución en la frecuencia

Presión arterial media 
cardiaca o un aumento del GC aumenta la PP.

2. Descenso de la complianza arterial (Ej. Aterosclerosis)

A partir de la PP se podría conocer latido a latido el volumen sistólico estimado, esta es la base de buena parte de los monitores de gasto cardiaco. Sabemos que:

Volumen sistólico estimado $=$ Presión de pulso * Factor $\mathrm{X}$

Donde

Factor X, variable hemodinámica depende del estado del sistema arterial.

\section{Contractilidad}

La onda arterial refleja el cambio de la presión a lo largo del tiempo (dP/dt). Cuanto más inclinada sea la rama anacrótica, teoricamente la $\mathrm{dP} / \mathrm{dt}$ sería reflejo de una mayor contractilidad cardiaca. Esta información ha sido empleada para valorar el inicio de inotrópicos pero lo cierto es que la relación entre la $\mathrm{dP} / \mathrm{dt}$ de la onda arterial y la contractilidad del ventrículo izquierdo es controvertida y bastante compleja (dependiente de la contractilidad, del flujo a través de la válvula aórtica, de la resistencia arterial periférica, de la presión diastólica y del patrón de activación eléctrico del ventrículo izquierdo). $\mathrm{La} \mathrm{dP} / \mathrm{dtmax}$ ventricular ocurre antes de la apertura de la válvula aórtica por lo que no se debería asumir que su medición a nivel periférico sea un fiel reflejo de lo que ocurre a nivel miocárdico (4). Sin embargo, parece que los cambios de $\mathrm{dP} / \mathrm{dt}$ a nivel ventricular y periférico si mantienen una mayor relación entre sí que los valores absolutos.

Volumen sistólico
El volumen sistólico podría dividirse en dos componentes; flujo sistólico $\mathrm{y}$ diastólico.

Se ha sugerido que el VS puede calcularse a partir de la morfología de la onda de pulso a través de la siguiente ecuación:

$\mathrm{VS}=K \times \mathrm{Pmd}(1+\mathrm{As} / \mathrm{Ad})$ donde;

VS = volúmen sistólico

$\mathrm{K}=$ constante que depende de la resistencia vascular, la distensibilidad de los vasos y de la impedancia aórtica.

Pmd $=$ presión de distensión media al final de la sístole

As = área bajo la curva sistólica.

$\mathrm{Ad}=$ área bajo la curva diastólica.

Entre las críticas a esta fórmula encontramos que asume que no se produce flujo retrógrado a lo largo del ciclo cardiaco (no podría aplicarse en caso de insuficiencia aórtica), y que no aborda el efecto sobre la onda de presión de las ondas de reflexión.

\section{Conclusión}

El análisis de la onda de pulso arterial, frecuentemente monitorizada en anestesia y cuidados intensivos, permite al clínico obtener una información valiosa del sistema cardiovascular latido a latido. Su correcta interpretación puede ser de gran utilidad a la hora de tomar decisiones clínicas

\section{Bibliografía}

1. Thiele RH, Durieux ME. Arterial Waveform Analysis for the Anesthesiologist: past, present and future concepts. Anesth Analg. 2011;113(4):766-76. ( PubMed) 
2. Chantler PD, Lakatta EG. Arterialventricular coupling with aging and disease. Front Physiol. 2012;3:909. (PubMed)

3. O'Rourke MF TM. Vascular impedance of the femoral bed. Circ Res. 1966;18:126-39. (PMC)

4. Wallace AG, Skinner NS Jr, Mitchell JH. Hemodynamic determinants of the maximal rate of rise of left ventricular pressure. Am J Physiol. 1963;205:30-6. (ㄹbMed)
Correspondencia al autor

Daniel Paz Martín

dpaz@anestesiar.org

MD PhD EDAIC EDIC. Servicio Anestesiología y Reanimación.

Complejo Hospitalario de Toledo.

Sección de Cuidados Intensivos de la SEDAR.

Aceptado para el blog en enero de 2020. 